\title{
Eosinophil pathogenicity mechanisms and therapeutics in neuromyelitis optica
}

\author{
Hua Zhang and A.S. Verkman \\ Departments of Medicine and Physiology, UCSF, San Francisco, California, USA.
}

\begin{abstract}
Eosinophils are abundant in inflammatory demyelinating lesions in neuromyelitis optica (NMO). We used cell culture, ex vivo spinal cord slices, and in vivo mouse models of NMO to investigate the role of eosinophils in NMO pathogenesis and the therapeutic potential of eosinophil inhibitors. Eosinophils cultured from mouse bone marrow produced antibody-dependent cell-mediated cytotoxicity (ADCC) in cell cultures expressing aquaporin-4 in the presence of NMO autoantibody (NMO-IgG). In the presence of complement, eosinophils greatly increased cell killing by a complement-dependent cell-mediated cytotoxicity (CDCC) mechanism. NMO pathology was produced in NMO-IgG-treated spinal cord slice cultures by inclusion of eosinophils or their granule toxins. The second-generation antihistamines cetirizine and ketotifen, which have eosinophil-stabilizing actions, greatly reduced NMO-IgG/eosinophil-dependent cytotoxicity and NMO pathology. In live mice, demyelinating NMO lesions produced by continuous intracerebral injection of NMOIgG and complement showed marked eosinophil infiltration. Lesion severity was increased in transgenic hypereosinophilic mice. Lesion severity was reduced in mice made hypoeosinophilic by anti-IL-5 antibody or by gene deletion, and in normal mice receiving cetirizine orally. Our results implicate the involvement of eosinophils in NMO pathogenesis by ADCC and CDCC mechanisms and suggest the therapeutic utility of approved eosinophil-stabilizing drugs.
\end{abstract}

\section{Introduction}

Neuromyelitis optica (NMO) is an inflammatory demyelinating disease that primarily affects the spinal cord and optic nerves, leading to paralysis and visual impairment $(1,2)$. Serum autoantibodies against astrocyte water channel aquaporin-4 (AQP4), called NMO immunoglobulin $\mathrm{G}$ (NMO-IgG), are present in most NMO patients and are believed to be pathogenic (3-5). Human NMO lesions show marked cellular infiltration with eosinophils, neutrophils, and macrophages, loss of astrocyte AQP4 and glial fibrillary acidic protein, perivascular deposition of activated complement, and demyelination (6-9). Current NMO therapies include immunosuppression, plasma exchange and $\mathrm{B}$ cell depletion $(10,11)$.

Eosinophil infiltration is a prominent feature in NMO lesions but not in other demyelinating diseases including multiple sclerosis $(2,7)$. Eosinophils are also found in cerebrospinal fluid in NMO (9). They can damage cells by the release of toxins contained in intracellular granules, including eosinophilic granule major basic protein (MBPe), eosinophil cationic protein (ECP), eosinophil peroxidase, and eosinophil-derived neurotoxin (12). Eosinophil degranulation can be triggered by immunoglobulin binding to $\mathrm{Fc}$ receptors and by soluble effectors such as complement anaphylotoxins C3a and C5a, chemokines, and lipid mediators (13). Differentiation and maturation of eosinophils in bone marrow is under the control of transcription factor GATA-1, as well as IL-3, IL-5, and GM-CSF (12). Eosinophils are normally present in the gastrointestinal tract, as well as in the thymus, mammary gland, and uterus. Eosinophilia and exaggerated eosinophil responses occur in some infections, asthma, hypereosinophilic syndrome, eosinophilic gastrointestinal diseases, and certain tumors (14). Eosinophil-based therapies target eosinophil production or eosinophil-derived products, which broadly include glucocorticoids,

Conflict of interest: The authors have declared that no conflict of interest exists. Citation for this article: J Clin Invest. 2013;123(5):2306-2316. doi:10.1172/JCI67554. myelosuppressive drugs, leukotriene antagonists and inhibitors, some second-generation antihistamines, cromoglycate, tyrosine kinase inhibitors, IFN- $\alpha$, and anti-IL-5 antibodies $(13,14)$.

Here, we utilize mouse models of NMO, including ex vivo spinal cord slice cultures (15) and a new in vivo intracerebral infusion model, as well as cell cultures, to investigate the mechanisms of eosinophil-dependent NMO pathology. We report evidence of antibody-dependent cell-mediated cytotoxicity (ADCC) and complement-dependent cell-mediated cytotoxicity (CDCC), in which eosinophil toxins secreted upon degranulation injure target cells. We also show protection against eosinophil-dependent NMO pathology by small-molecule inhibitors of eosinophil degranulation, including 2 widely used second-generation antihistamines.

\section{Results}

Eosinophils produce NMO-IgG-dependent cytotoxicity. We performed in vitro experiments with murine eosinophils cultured from bone marrow. Cultures were treated with SCF and FLT3 ligand (FLT3-L) for 4 days to promote eosinophil progenitor cell growth, followed by IL-5 for 10 days to simulate eosinophil proliferation, and then GM-CSF for 24 hours before experiments to induce Fc receptor surface expression $(16,17)$. We produced a nearly pure suspension of eosinophils after 14 to 16 days in culture, showing cytoplasmic MBPe expression (Figure 1A).

The ability of eosinophils to produce NMO-IgG-dependent ADCC was studied after 3 hours' incubation of AQP4-expressing $\mathrm{CHO}$ cell cultures with NMO-IgG and eosinophils. The percentage and density of live cells were quantified by live/dead (green/ red) cell staining (Figure 1B). Substantial cell death was produced when NMO-IgG and eosinophils were present. Little cell death was seen in untreated (control) cells, in cells incubated with NMO-IgG or eosinophils alone, or in AQP4-null cells incubated with NMOIgG and eosinophils. Also, little cell death was seen when NMOIgG was replaced by a mutated NMO-IgG lacking ADCC effector 
A

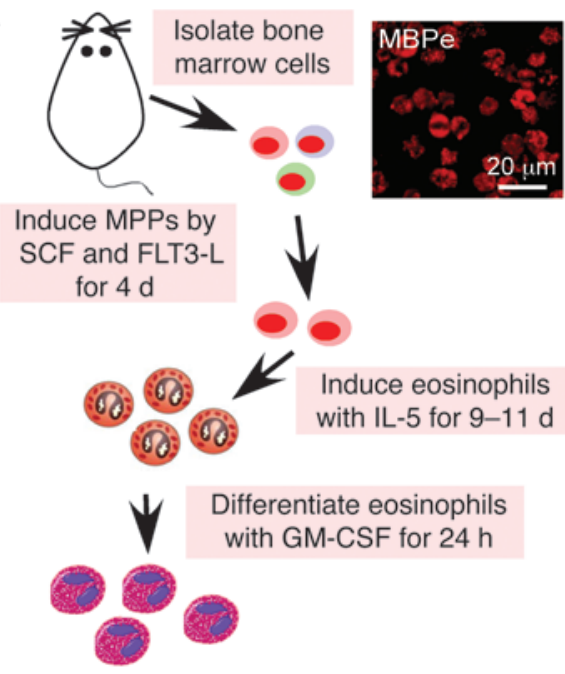

C

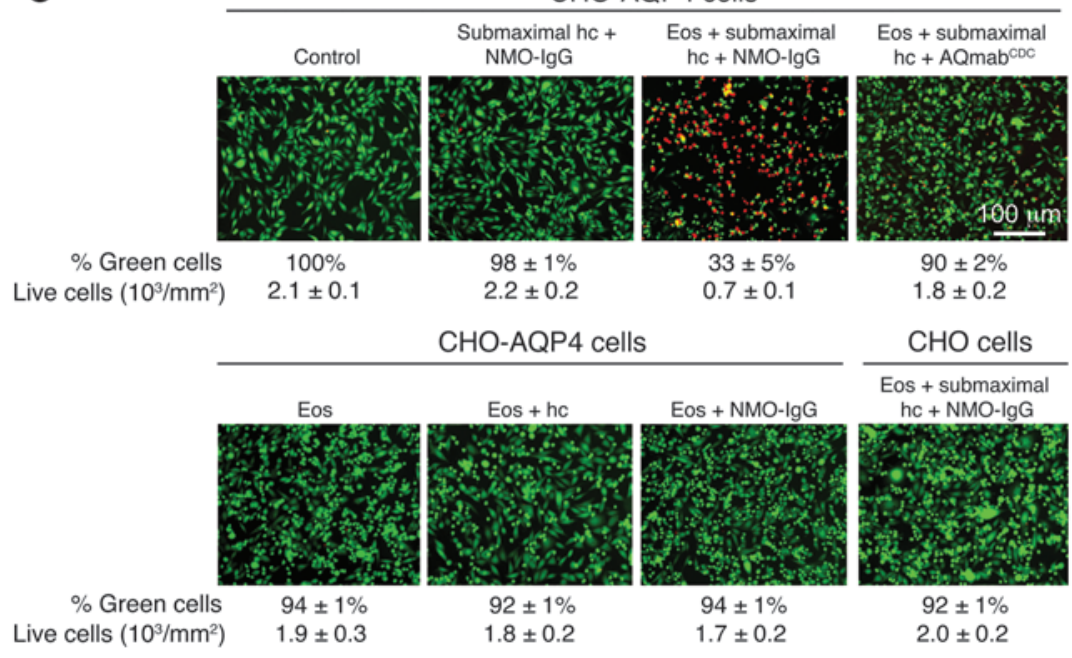

B

Live/dead cell staining

CHO-AQP4 cells
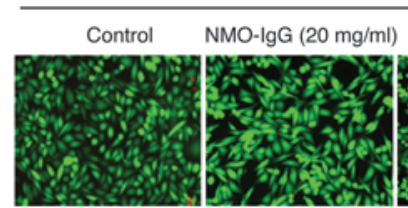

Eos Eos + NMO-IgG

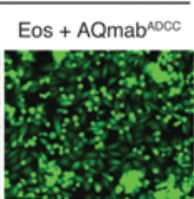

CHO cells
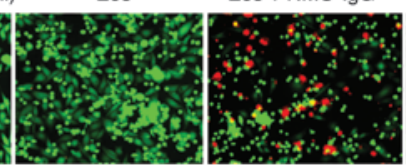

Eos + NMO-IgG

$\%$ Green cells Live cells $\left(10^{3} / \mathrm{mm}^{2}\right)$

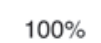

$2.3 \pm 0.1$

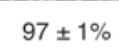

$97 \pm 1 \%$
$2.0 \pm 0.2$
$90 \pm 1 \%$ $90 \pm 1 \%$
$1.8 \pm 0.2$

\begin{abstract}
$25 \pm 3 \%$
\end{abstract}
$25 \pm 3 \%$
$0.5 \pm 0.3$
$89 \pm 1 \%$

$1.8 \pm 0.2$

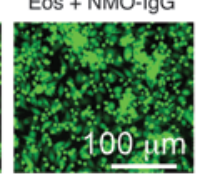

$92 \pm 1 \%$

$2.2 \pm 0.1$

\section{Figure 1}

Eosinophils produce NMO-lgG-dependent ADCC and CDCC in cell cultures. (A) Culture and characterization of eosinophils from murine bone marrow. Culture method shown at the left and MBPe immunofluorescence shown in the micrograph. Scale bar: 20 um. (B) ADCC. AQP4-

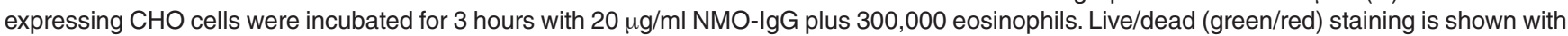
percentage and density of live cells given below the micrographs (SEM, $n=10$ ). Controls included untreated cultures, NMO-IgG or eosinophils alone, AQmab ${ }^{A D C C}$ plus eosinophils, and NMO-IgG plus eosinophils in nontransfected (AQP4-null) cells. Scale bar: $100 \mu \mathrm{m}$. (C) CDCC. Cells were incubated for 60 minutes with submaximal $(5 \mu \mathrm{g} / \mathrm{ml})$ NMO-lgG, $1 \%$ hc, and 100,000 eosinophils, with controls indicated. Percentage of dead cells quantified as in B (SEM, $n=10)$. Scale bar: $100 \mu \mathrm{m}$. Experiments were replicated 3 times. Eos, eosinophils; MPPs, multipotent progenitors.

function (AQmab $\left.{ }^{A D C C}\right)$. Eosinophils can thus produce ADCC in the presence of NMO-IgG.

The ability of eosinophils to produce CDCC was measured similarly, except that cells were incubated with submaximal NMO-IgG and human complement (hc) to produce minimal cell death in the absence of eosinophils (Figure 1C). The inclusion of eosinophils greatly increased cell killing. The various controls indicate that eosinophil-dependent CDCC requires NMO-IgG, complement, and AQP4.

Eosinophils exacerbate NMO lesions in spinal cord slice cultures. The ability of eosinophils to produce NMO pathology by ADCC and CDCC mechanisms was investigated using an ex vivo spinal cord slice culture model (15). Vibratome-cut spinal cord slices from 7-day-old mice were cultured for 7 days, then NMO-IgG and eosinophils were added for 1 day, after which slices were immunostained for markers of NMO pathology. Figure 2A shows immunofluorescence staining for AQP4, glial fibrillary acidic protein (GFAP), myelin basic protein (MBP), and ionized calcium-binding adaptor molecule 1 (Iba1, activated microglia), with lesion scores (0: no pathology; 4: severe pathology) summarized at the bottom. We observed a loss of AQP4 and GFAP and increased Iba1 in slices exposed to NMO-
IgG and eosinophils and little loss of MBP immunofluorescence. We also saw little pathology in control slices or in slices incubated with NMO-IgG or eosinophils alone, or with AQmab ${ }^{\mathrm{ADCC}}$ and eosinophils. We saw little pathology in slices from AQP4-null mice incubated with NMO-IgG and eosinophils. Eosinophils can thus produce NMO-like pathology in spinal cord slice cultures by an ADCC mechanism. We postulated that toxic factors elaborated by degranulating eosinophils may be responsible for the cytotoxicity. To induce eosinophil degranulation, we added platelet-activating factor (PAF) to slice cultures. Figure $2 \mathrm{~B}$ shows a marked loss of GFAP and AQP4 in slice cultures incubated with eosinophils in the presence of PAF (in the absence of NMO-IgG), but not in those incubated with eosinophils or PAF alone.

Similar studies done in parallel with those in Figure 2A indicated the involvement of eosinophils in CDCC, in which spinal cord slice cultures were exposed to submaximal NMO-IgG and complement (Figure 3A). Incubation with eosinophils greatly increased NMO pathology. Control studies showed that eosinophil-dependent pathology was dependent on NMO-IgG, complement, and AQP4 expression. Replacement of NMO-IgG by a mutated NMO-IgG lacking complement-dependent cytotoxicity (CDC) effector function 
A
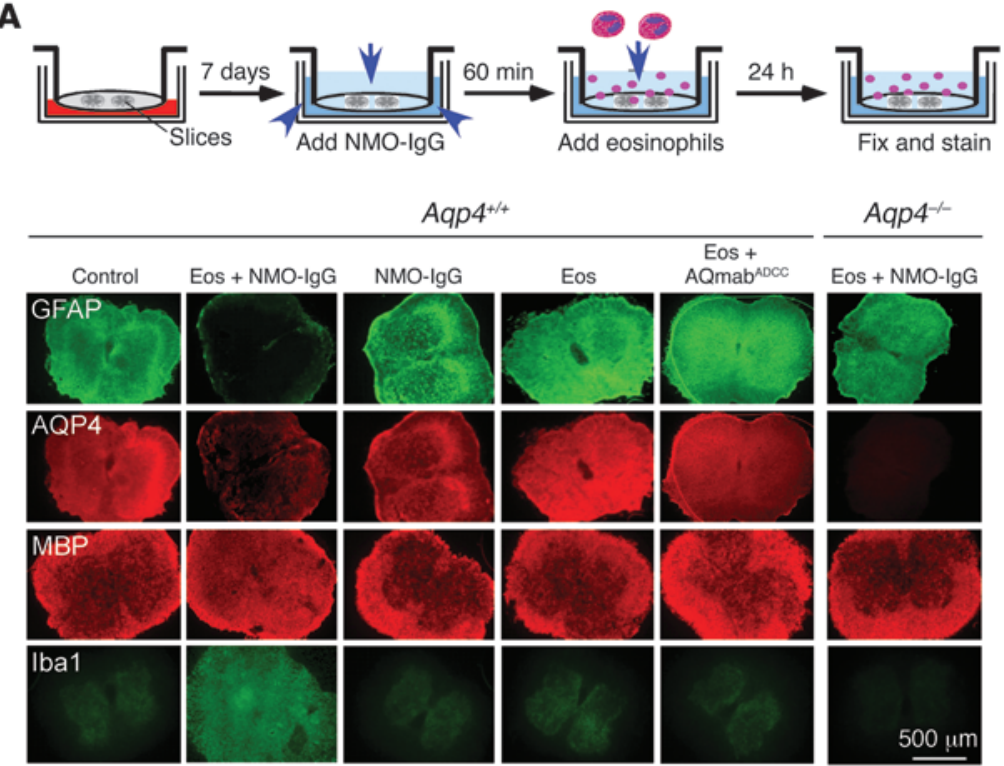

$\star$

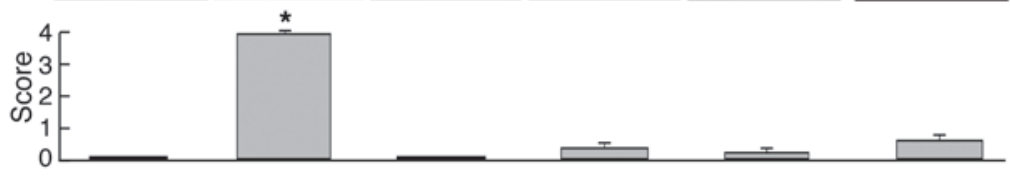

\section{Figure 2}

Eosinophils produce NMO-IgG-dependent pathology by an ADCC mechanism in ex vivo spinal cord slice cultures. (A) Vibratome-cut spinal cord slices from mouse were cultured on a porous support for 7 days, followed by the addition of NMO-IgG and/or cells for 1 day, and then stained for AQP4, GFAP, MBP, and Iba1. Immunofluorescence of spinal cord slices (from Aqp $4^{+/+}$and Aqp4 $4^{-/-}$mice) were incubated as indicated $(20 \mu \mathrm{g} / \mathrm{ml}$ NMO-IgG or AQmab ${ }^{A D C C}, 10^{7}$ eosinophils). Pathology scores are summarized at the bottom (SEM; 6-11 slices per condition; ${ }^{*} P<0.001$ versus the control group; 1-way ANOVA). Scale bar: $500 \mu \mathrm{m}$. (B) Eosinophil degranulation by PAF produces NMO-like pathology ( $10^{7}$ eosinophils, 10 $\mu \mathrm{g} / \mathrm{ml} \mathrm{PAF}$ ) (SEM; 5 slices per condition; $\left.{ }^{\star} P<0.01\right)$. Scale bar: $500 \mu \mathrm{m}$. Experiments were replicated 3 times.

\section{B}
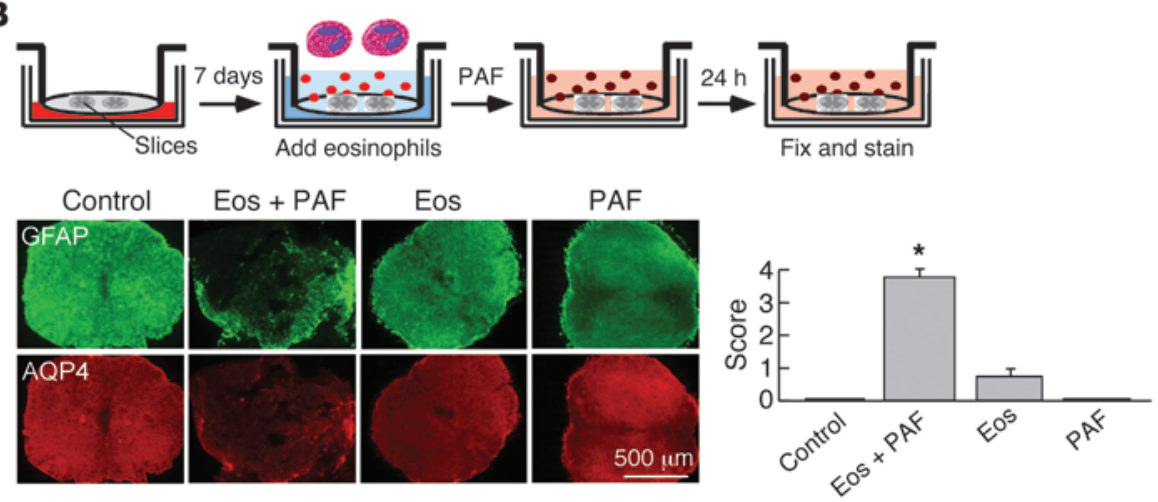

(AQmab ${ }^{\mathrm{CDC}}$ ) indicated that the eosinophil-dependent pathology was complement dependent (C1q-binding dependent), implicating a CDCC mechanism. C5a is a complement anaphylotoxin that promotes eosinophil chemotaxis and degranulation (18). Figure 3B shows a marked loss of GFAP and AQP4 in slice culture incubated with eosinophils in the presence of $\mathrm{C} 5 \mathrm{a}$ (in the absence of NMO$\mathrm{IgG}$ ), but not in culture incubated with eosinophils or C5a alone. We also found that the effect of eosinophils could be mimicked by the addition of purified human ECP, an eosinophil granule toxin, together with submaximal NMO-IgG and complement (Figure 3C).

Eosinophil-dependent NMO pathology in an in vivo mouse model of NMO. Prior passive-transfer mouse models of NMO involving intracerebral brain injection of NMO-IgG and complement produced NMO-like pathology, but without eosinophil infiltration $(19,20)$. After testing multiple variations of existing models, including eotaxin and IL-5 infusion together with NMO-IgG and complement administration, we found that human-like NMO pathology could be produced by 3-day continuous intracerebral infusion of NMO-IgG and he (Figure 4A). Immunofluorescence showed lesions with astrocyte destruction, loss of AQP4, GFAP, and excitatory amino acid transporter 1 (EAAT1) immunoreactivity, as well as leukocyte infiltration and demyelination (Figure 4B). We did not observe lesions in identically treated $A q \mathrm{p}^{4^{-/-}}$mice. Within the lesion core, there was near-complete loss of AQP4 and myelin, perivascular deposition of activated complement, and prominent inflammation with eosinophil and neutrophil infiltration (Figure 4C); in the lesion periphery there was reduced AQP4 and myelin without complement deposition, and prominent perivascular infiltration of eosinophils and neutrophils. Figure 4D summarizes the extent of AQP4 loss, showing little pathology in $A q p 4^{-/-}$mice or wild-type mice infused with NMO-IgG alone, or with control IgG with complement. Similar lesions were produced in mice infused continuously for 3 days with purified IgG from human NMO sera together with complement. Interestingly, after 1-day infusion with NMO-IgG and complement, the lesions contained many neutrophils but few eosinophils (Figure 4E).

Several types of studies were conducted to demonstrate eosinophil-dependent NMO pathology in vivo. In an initial set of 
A
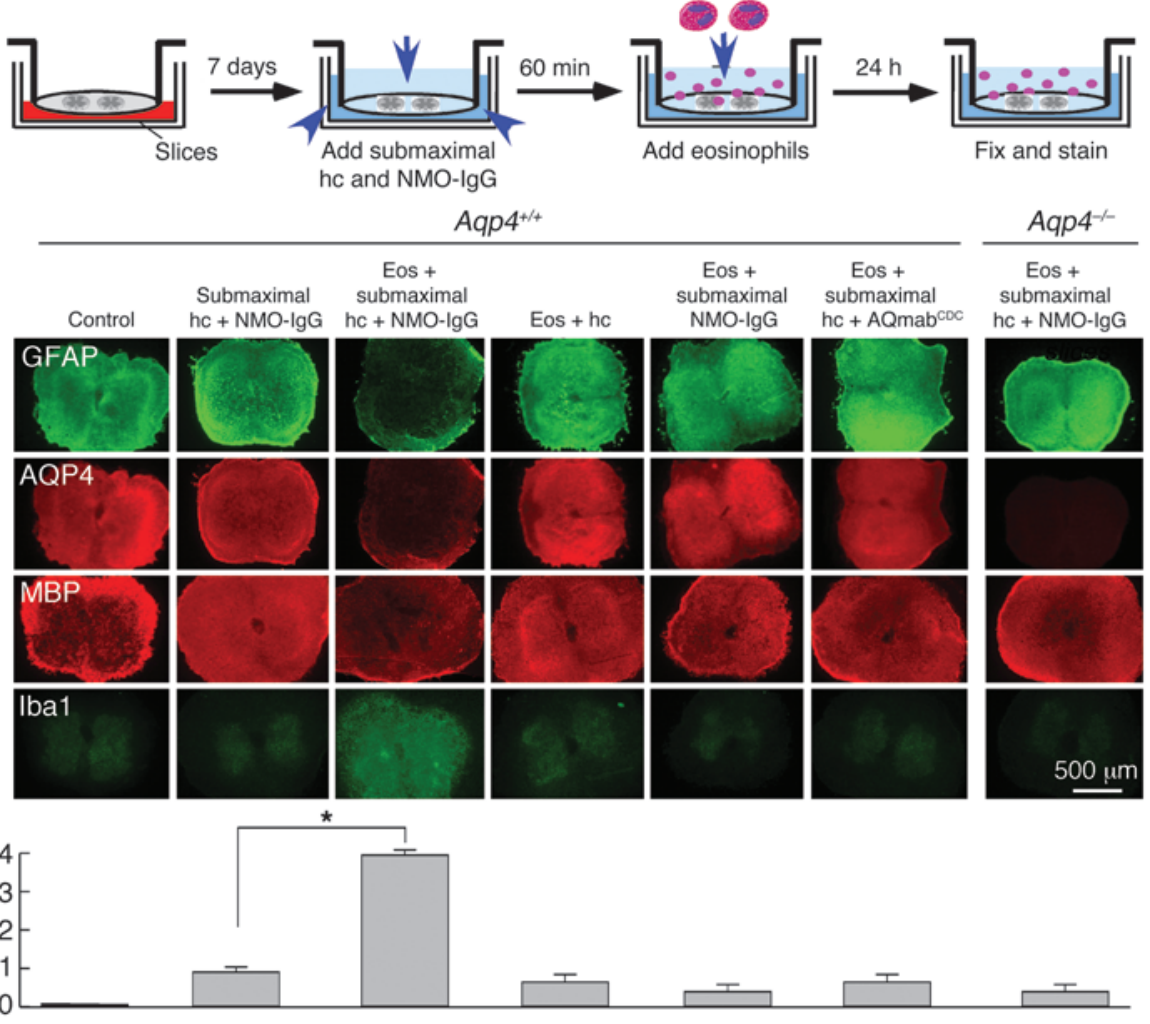

B
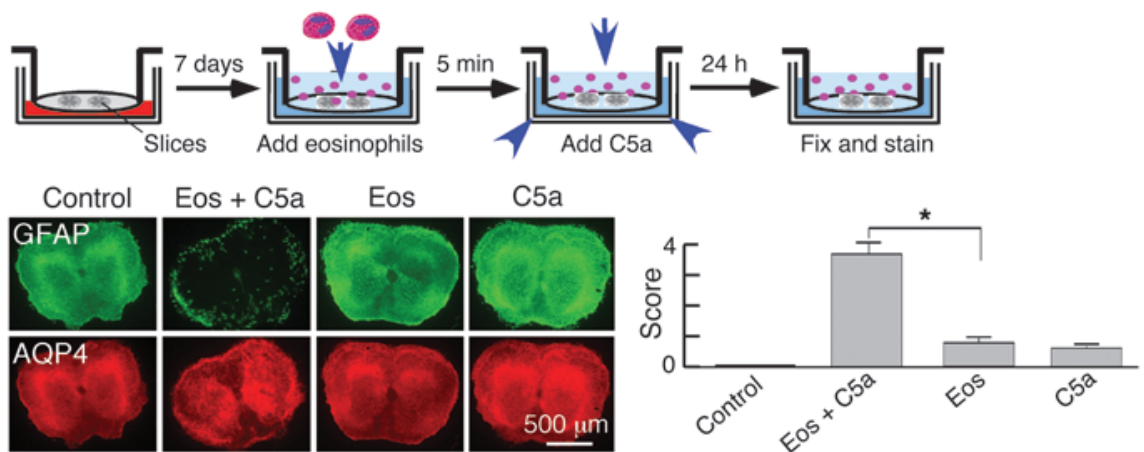

C
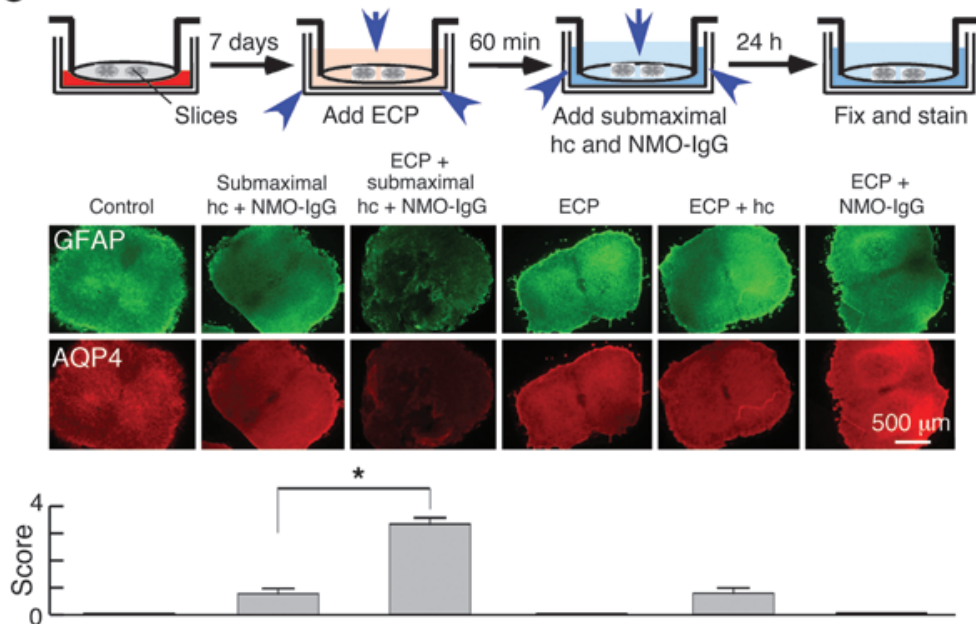

\section{Figure 3}

Eosinophils produce NMO-IgG-dependent pathology by a CDCC mechanism in ex vivo spinal cord slice cultures. (A) Spinal cord slice cultures prepared as in Figure 2 were incubated with submaximal NMO$\mathrm{IgG}$ and hc as indicated $(5 \mu \mathrm{g} / \mathrm{ml}$ NMOIgG or AQmabCDC, $3 \times 10^{6}$ eosinophils, $5 \% \mathrm{hc}$ ). The experiments were conducted in parallel with those in Figure $2 \mathrm{~A}$ using the same control slice group (SEM; 8-11 slices per condition; ${ }^{*} P<0.001$ versus the control group; 1-way ANOVA). Scale bar: $500 \mu \mathrm{m}$. (B) Eosinophil degranulation by recombinant human $\mathrm{C} 5$ a produces NMOlike pathology $\left(10^{7}\right.$ eosinophils pretreated with $5 \mu \mathrm{g} / \mathrm{ml}$ cytochalasin B, $30 \mathrm{ng} / \mathrm{ml} \mathrm{C5a)}$ (SEM; 5 slices per condition; ${ }^{\star} P<0.01$ ). Scale bar: $500 \mu \mathrm{m}$. (C) Eosinophil toxin ECP produces NMO-like pathology (5 $\mu \mathrm{g} / \mathrm{ml} \mathrm{NMO}-\mathrm{lgG}, 5 \% \mathrm{hc}, 300 \mathrm{ng} / \mathrm{ml}$ ECP) (SEM; 4 slices per condition; ${ }^{\star} P<0.01$ ). Scale bar: $500 \mu \mathrm{m}$. Experiments were replicated 3 times. 
A

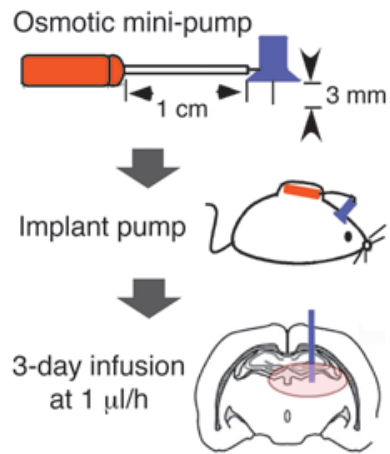

B

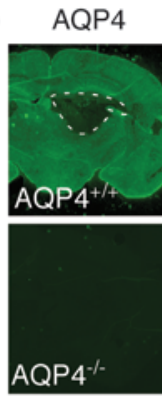

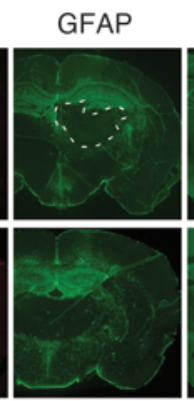
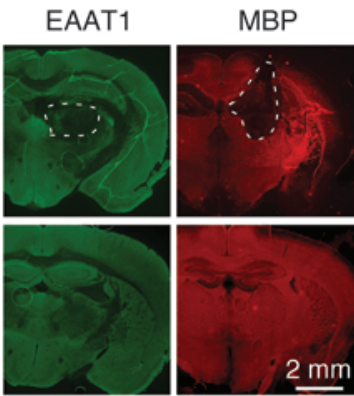

CD45

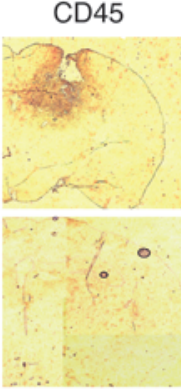

C
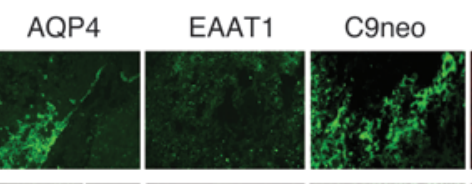

MBP
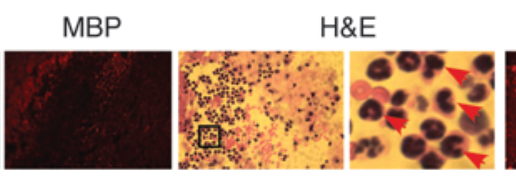

Siglec F

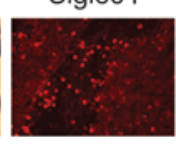

Ly6G
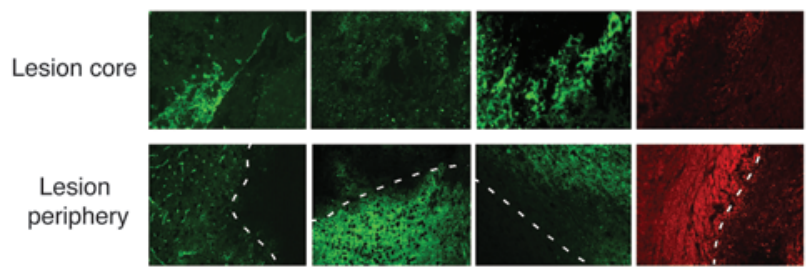

periphery
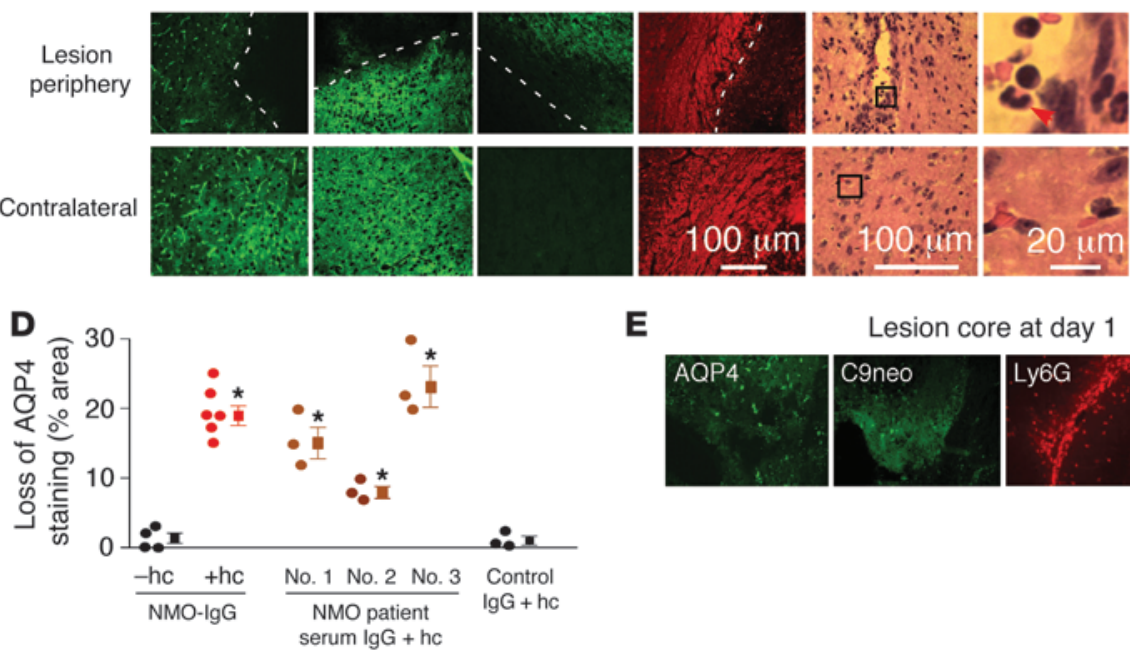

E

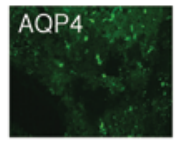

Lesion core at day 1

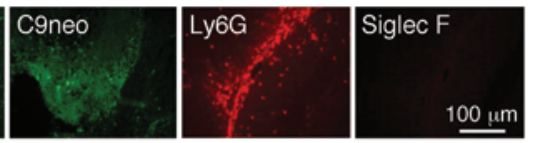

\section{Figure 4}

In vivo mouse model of NMO with prominent eosinophil infiltration in lesions. (A) Mice were administered NMO-IgG and hc by 3-day continuous intracerebral injection using an implanted minipump. (B) Immunofluorescence staining of brain sections after 3-day infusion with NMO-IgG (3.3 $\mu \mathrm{g} /$ day) and complement (16.7 $\mu \mathrm{l} /$ day) stained for astrocyte markers AQP4, GFAP, and EAAT1, myelin marker MBP, and leukocyte marker CD45, in Aqp4 $4^{+/}$and $\mathrm{Aqp4}^{-/-}$mice. White dotted lines demarcate the area of staining loss. Scale bar: $2 \mathrm{~mm}$. (C) Higher magnification showing AQP4 and EAAT1, MBP, activated complement marker C9neo, markers of eosinophils (Siglec F) and neutrophils (Ly6G), and H\&E staining in the lesion core, periphery, and contralateral side. Scale bars: $100 \mu \mathrm{m}$. Arrowhead indicates eosinophils in high magnificent H\&E staining. Scale bar: $20 \mu \mathrm{m}$. (D) Percentage of area of loss of AQP4 staining (SEM; 3-6 mice per group; ${ }^{*} P<0.05$ ). Mice were infused for 3 days as in $\mathrm{B}$ with NMO-IgG alone, control IgG plus complement, or IgG (66 $\mathrm{gg} /$ day) purified from 3 different NMO sera. (E) Immunofluorescence of AQP4, C9neo, Siglec F, and Ly6G after 1 day of minipump infusion (representative of 3 mice). Scale bar: $100 \mu \mathrm{m}$.

studies, lesions were compared in control mice receiving NMOIgG and complement for 3 days with mice that were pretreated with anti-IL-5 to deplete eosinophils (21). Figure 5A summarizes peripheral eosinophil and neutrophil counts, showing the efficacy of the anti-IL-5 antibody. Figure 5B shows reduced lesion size and eosinophil infiltration in the anti-IL-5 antibody-treated mice. In a second set of studies, in order to isolate the effects of eosinophils versus neutrophils, we compared NMO lesions produced by NMOIgG and complement in mice made neutropenic with anti-Ly6G antibody (20), without versus with anti-IL-5 antibody treatment. Treatment with anti-Ly6G antibody significantly reduced lesion size, which was further reduced by anti-IL-5 treatment (Figure $5 \mathrm{C})$. Interestingly, in the neutropenic mice, the number of eosinophils in the lesion increased, particularly around blood vessels. In a third set of experiments, we tested the consequences of peripheral hypereosinophilia. Following infusion for 3 days with submaximal NMO-IgG and complement, lesions in transgenic hypereosinophilic (IL-5 Tg) mice showed marked eosinophil infiltration, with larger lesions compared with those in the control (normo-eosinophilic) mice (Figure 5D). Finally, studies in $\Delta d b l$ Gata1 mice, which lack eosinophils due to Gata1 promoter mutation, showed reduced lesion size (Figure 5E).

Eosinophil inhibitors reduce NMO-IgG-dependent pathology. We tested the possibility that compounds known to inhibit eosinophil degranulation would reduce NMO pathology. Figure 6A (left) shows in vitro inhibition of eosinophil degranulation, as assayed by EPO release, by cetirizine, a second-generation antihistamine, and IBMX, a phosphodiesterase inhibitor. The $\mathrm{IC}_{50}$ 
A

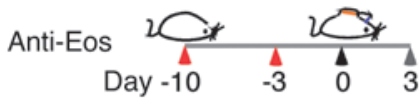

Anti- $\mathrm{N} \phi$

Anti-Eos

$+\operatorname{anti-N\phi } \begin{array}{rrrrr}-10 & -3 & -10 & 10 & 1\end{array}$

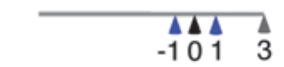

$\Delta$ Inject anti-N $\phi$ Ab $\quad \Delta$ Collect brain tissue

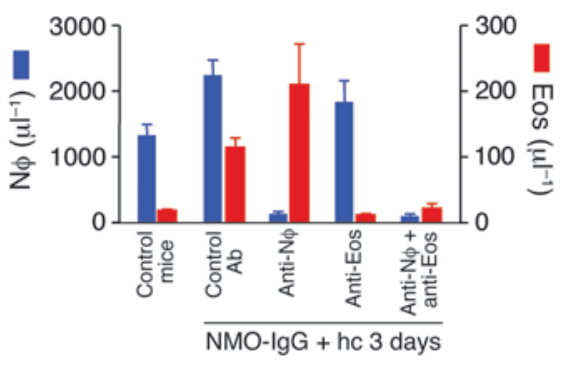

$\Delta$ Inject anti-Eos Ab $\Delta$ Implant pump

B
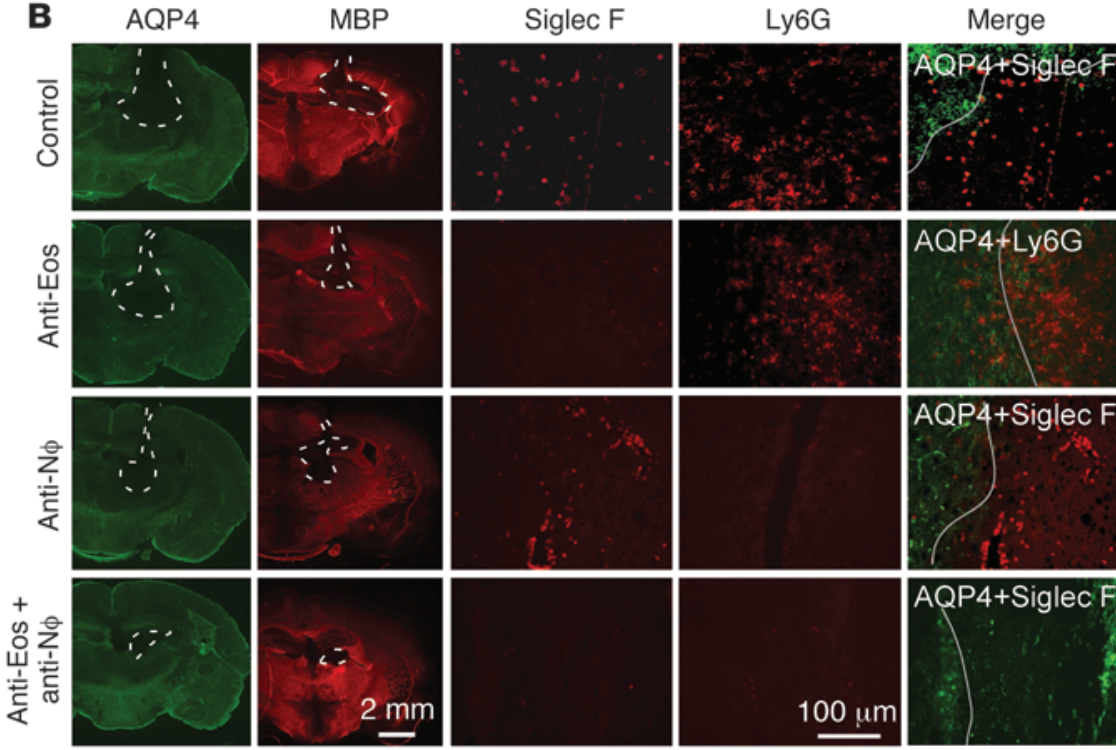

C
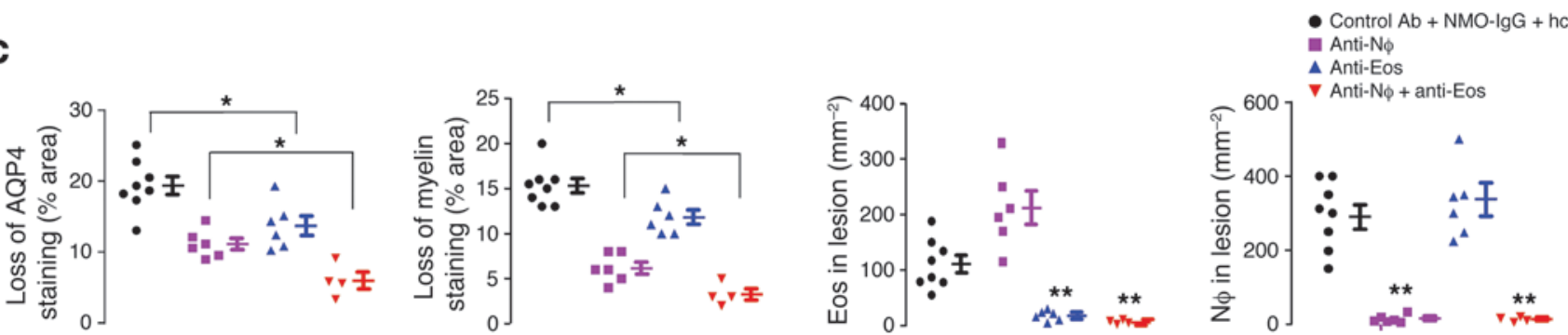
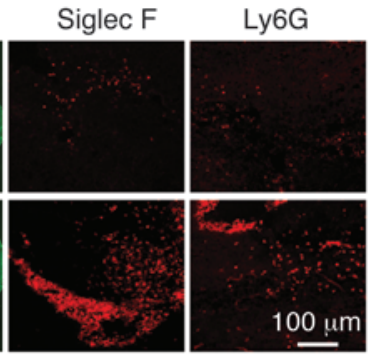

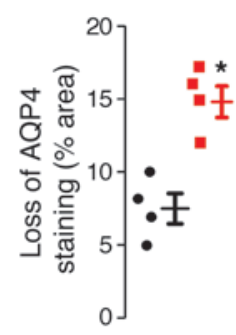

- Control mice - IL-5 Tg mice
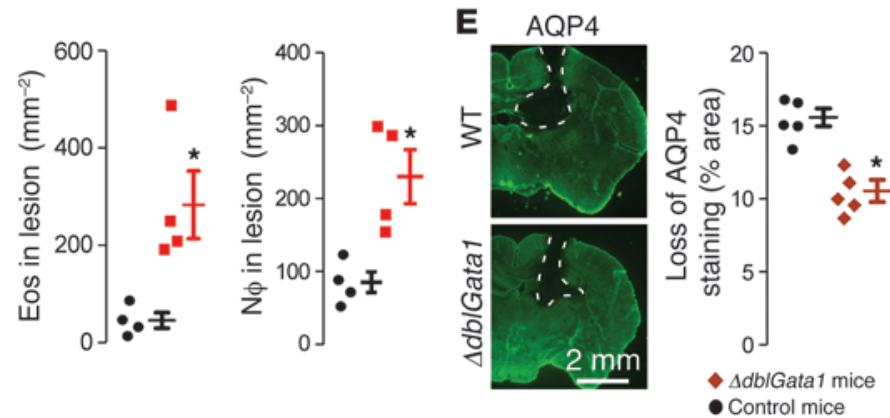

Figure 5

Eosinophil-dependent NMO pathology in mice. (A) Mice were intraperitoneally administered anti-IL-5 or anti-Ly6G antibodies, alone or together, to deplete eosinophils and/or neutrophils, followed by a 3-day NMO-lgG/hc infusion (top). Peripheral neutrophil ( $\mathrm{N} \phi)$ and eosinophil counts in control and treated mice (bottom) (SEM; 5 mice per group; $P<0.001$ ). (B) Mice were infused for 3 days with $3.3 \mu \mathrm{g} / \mathrm{day} \mathrm{NMO-lgG}$ and $16.7 \mu \mathrm{l} /$ day hc, and sacrificed on day 3. Immunofluorescence staining of AQP4 and MBP (scale bar: $2 \mathrm{~mm}$ ); Siglec F and Ly6G (scale bar: $100 \mu \mathrm{m}$ ). (C) Lesion severity assessed by loss of AQP4, myelin, and numbers of eosinophils and neutrophils in lesions (SEM; 4-8 mice per group; ${ }^{*} P<0.05$; $\left.{ }^{* *} P<0.01\right)$. (D) Control and hypereosinophilic IL-5 Tg mice were infused for 3 days with NMO-IgG (3.3 $\mu \mathrm{g} /$ day) and submaximal hc (3.4 $\left.\mu \mathrm{l} / \mathrm{day}\right)$. Immunofluorescence staining of AQP4 (scale bar: $2 \mathrm{~mm}$ ), Siglec F, and Ly6G (scale bar: $100 \mu \mathrm{m}$ ). Summary of lesion scores (SEM; 4 mice per group; $\left.{ }^{*} P<0.05\right)$. (E) Control and Tg hypoeosinophilic $\Delta d b / G a t a 1$ mice were infused for 3 days with NMO-lgG (3.3 $\left.\mu \mathrm{g} / \mathrm{day}\right)$ and hc (16.7 $\left.\mu \mathrm{l} / \mathrm{day}\right)$. AQP4 immunofluorescence and loss of AQP4 immunofluorescence are shown (SEM; 5 mice per group; $\left.{ }^{\star} P<0.05\right)$. Scale bar: 2 mm.

for cetirizine inhibition was approximately $1 \mu \mathrm{M}$. Figure $6 \mathrm{~A}$ (center) shows relatively lower potency of other eosinophil stabilizers including theophylline, ketotifen, cromolyn, and loratadine. Control studies showed that these compounds did not protect against CDC in AQP4-expressing cells produced by NMO-IgG and complement in the absence of eosinophils (Figure 6A, right). Figure $6 \mathrm{~B}$ shows that cetirizine reduced eosinophil degranulation in AQP4-expressing cell cultures treated with NMO-IgG and eosinophils, with low EPO activity in control (eosinophil alone) measurements. We found protective effects of cetirizine, ketotifen, and IBMX for eosinophil-dependent NMO pathology in spinal cord slice cultures. Figure $6 \mathrm{C}$ shows significant protection in the eosinophil-dependent ADCC (top) and CDCC (bottom) models established in Figures 2 and 3. 


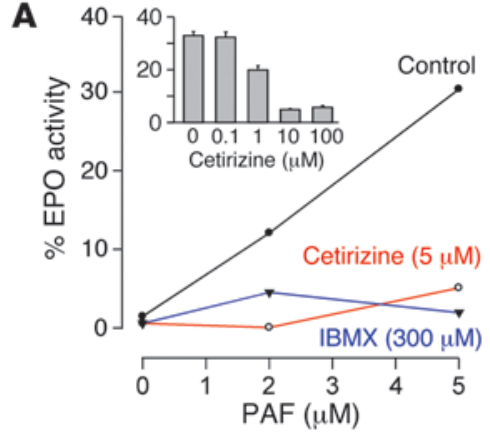

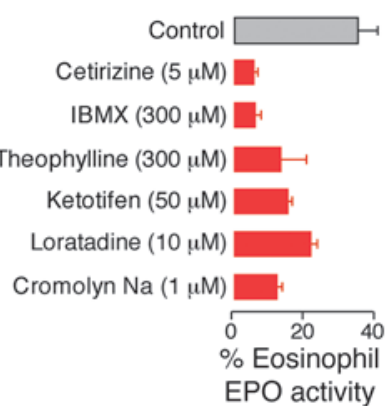

$\mathrm{c}+\mathrm{NMO}-\operatorname{lgG}$

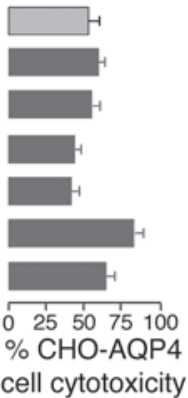

C
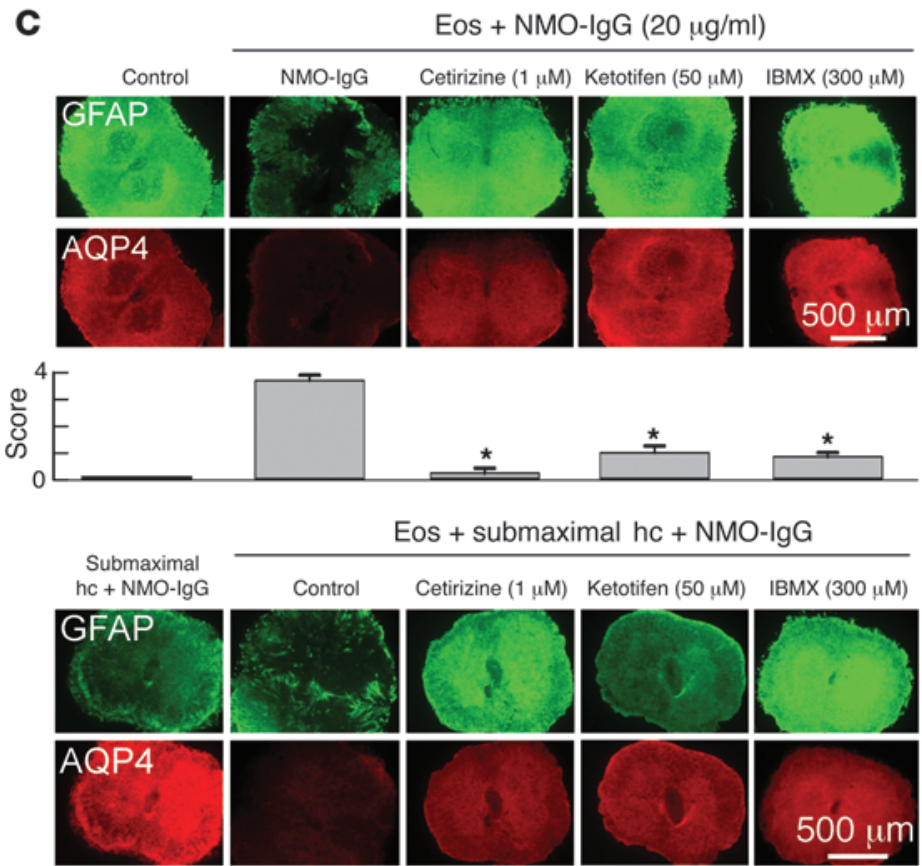

Eos + submaximal hc + NMO-lgG

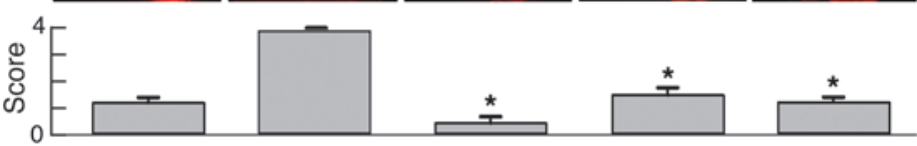

E<smiles>CNCCn1nccc1-c1ccccc1</smiles>

Mepyramine
Cetirizine $(1 \mu \mathrm{M}) \quad$ Ketotifen $(50 \mu \mathrm{M}) \quad$ IBMX $(300 \mu \mathrm{M})$
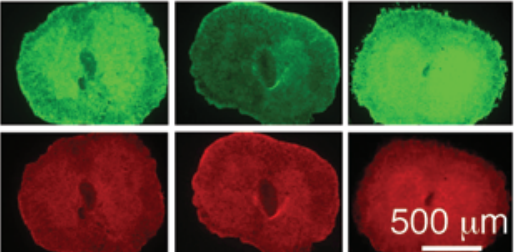

D

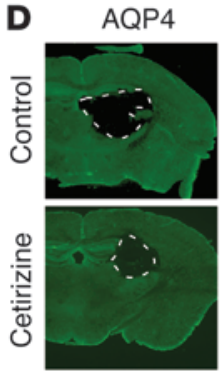

MBP
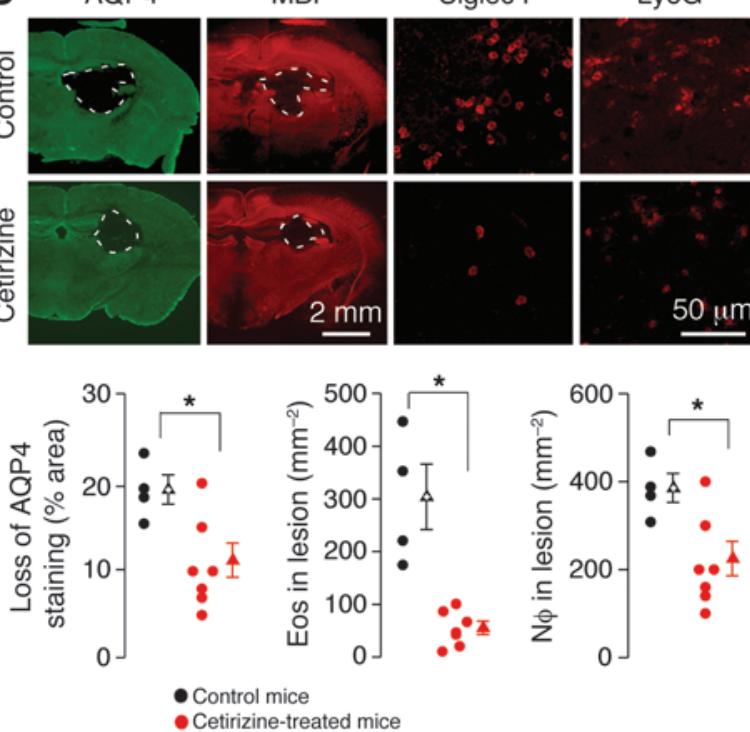

Cetirizine-treated mice
Siglec $\mathrm{F}$

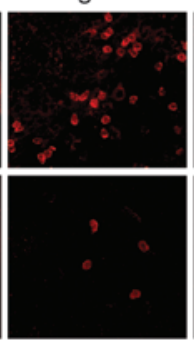

$50 \mu \mathrm{m}$

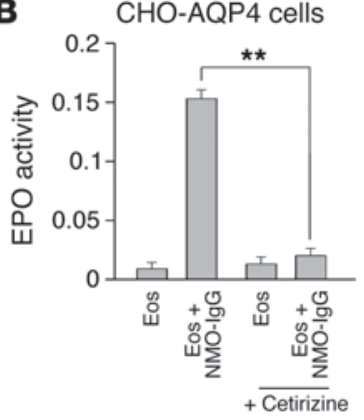

Ly6G

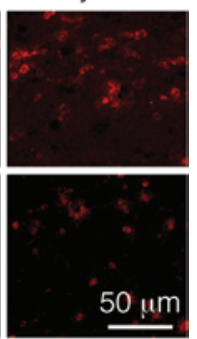

Figure 6

Small-molecule eosinophil inhibitors reduce NMO-IgG-dependent cytotoxicity and NMO lesions. (A) Reduced EPO release following 30 -minute incubation of eosinophils with $5 \mu \mathrm{M}$ PAF and indicated inhibitors (representative of 3 sets of experiments). Inset shows dose response for cetirizine (at $5 \mu \mathrm{M}$ PAF, $n=6$ ) (left). Summary of EPO release data (SEM; $n=6$ ) (center). Lack of compound effect on NMO-IgG-mediated CDC in AQP4-expressing $\mathrm{CHO}$ cells (SEM; $n=8$ ) (right). (B) EPO release into media for AQP4-expressing CHO cells treated for 1 hour with $10^{6}$ eos-

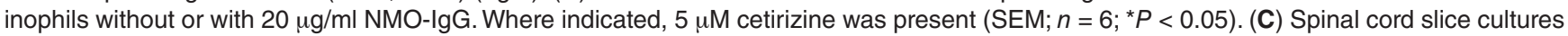
were exposed to NMO-IgG without complement (top, ADCC), or with complement (bottom, CDCC), and without or with eosinophils, as done in Figures 2 and 3. Where indicated, cetirizine $(1 \mu \mathrm{M})$, ketotifen $(50 \mu \mathrm{M})$, or IBMX $(300 \mu \mathrm{M})$ were present during the 24-hour incubation. Scale bars: $500 \mu \mathrm{m}$. (D) Intracerebral infusion was done (as in Figure 5) without versus with cetirizine administration (10 mg/kg b.i.d., 1 day prior to and during 3-day infusion). Immunofluorescence staining is shown on the top and lesion scores are summarized on the bottom (SEM; $n=4-6$; $\left.{ }^{*} P<0.05\right)$. Scale bars: $2 \mathrm{~mm} ; 50 \mu \mathrm{m}$. (E) Chemical structures of cetirizine and analogs (left). Summary of EPO release data (SEM; $\left.n=6\right)($ center). Summary of data from intracerebral infusion model (right) (each compound was given $10 \mathrm{mg} / \mathrm{kg}$ b.i.d.) (SEM; $n=3-7$ for each group; $\left.{ }^{*} P<0.05\right)$. 


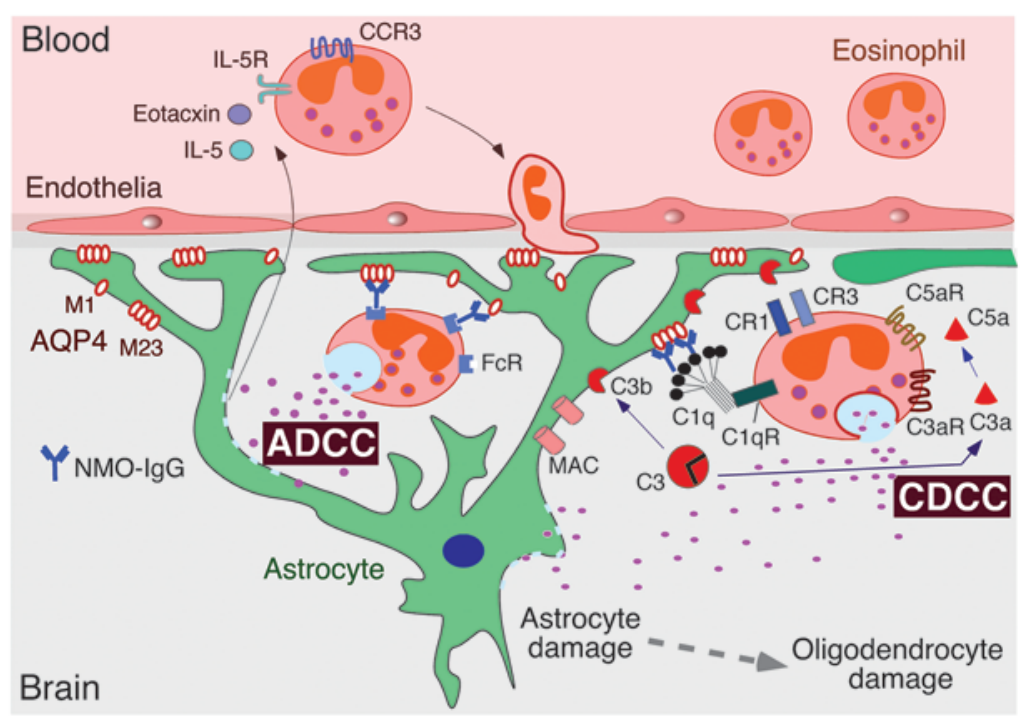

\section{Figure 7}

Proposed mechanism of eosinophil-dependent NMO pathogenesis. Eosinophils produce complement-independent and -dependent astrocyte damage. ADCC (left) involves binding of NMO-lgG to AQP4 on astrocytes, which causes eosinophil binding (involving Fcy receptors) and degranulation. The released granule toxins damage astrocytes. Astrocyte damage is amplified when complement is present by CDCC (right), which involves multiple mechanisms, including complement-dependent enhancement of eosinophil binding to AQP4-IgG and increased eosinophil accumulation and degranulation.
Cetirizine, which showed the greatest potency in vitro and ex vivo, was tested in mice in vivo. Mice were administered cetirizine $(10 \mathrm{mg} /$ $\mathrm{kg}$ b.i.d.) orally, as done previously in other disease models in mice (22), 1 day prior to and during the 3-day intracerebral infusion with NMO-IgG and complement. Figure 6D shows remarkably fewer eosinophils in lesions, with significantly reduced lesion severity.

Three first-generation antihistamine analogs of cetirizine, chlorphenamine, mepyramine, and hydroxyzine (Figure 6E, left) were also tested in this model. The analogs showed weak inhibition of eosinophil degranulation in vitro as assessed by EPO (Figure 6E, center), and no significant reduction of NMO lesion size in vivo (Figure 6E, right).

\section{Discussion}

The bulk of evidence supports an NMO pathogenic mechanism that involves NMO-IgG binding to AQP4 on astrocytes, which causes primary complement- and cell-dependent cytotoxicity and results in an inflammatory response involving cytokine release, blood-brain barrier disruption, and infiltration of granulocytes and macrophages $(2,4,5)$. The consequent oligodendrocytic injury produces demyelination and neuronal death, resulting in neurological deficit. The supramolecular assembly of AQP4 in orthogonal arrays of particles is crucial for NMO pathogenesis, as AQP4 aggregates enhance NMO-IgG binding (23) and present NMO-IgG clusters for efficient $\mathrm{C} 1 \mathrm{q}$ binding and consequent CDC (24). Several alternative NMO pathogenic mechanisms have been proposed but largely refuted, including astrocytic endocytosis of AQP4 and excitatory amino acid transporter-2, NMO-IgG inhibition of AQP4 water permeability, and cell membrane AQP4 clustering due to differential internalization of AQP4 M1 and M23 isoforms $(25,26)$. Elucidation of NMO pathogenesis is of clinical importance for the development of targeted, mechanism-based therapies, as current therapies, such as immunosuppression and B-cell depletion, are nonselective and of limited efficacy and can be associated with long-term adverse events.

Drug targeting of infiltrating granulocytes is a potential approach for NMO therapy. Eosinophils and neutrophils are the predominant infiltrating leukocytes, particularly in relatively early NMO lesions, with macrophages generally seen later $(6,19)$. Lym- phocytes and NK cells are rarely seen. We postulated that granulocyte toxins, which are released in response to various inflammatory stimuli, may participate in NMO pathogenesis. We reported recently that neutrophils and neutrophil elastase exacerbate NMO pathology in spinal cord slice cultures exposed to NMO-IgG and complement, and that the neutrophil elastase inhibitor Sivelestat largely prevented the neutrophil-dependent pathology (15). NMO pathology was greatly exacerbated in neutrophilic mice administered NMO-IgG and complement by intraparenchymal injection, and was reduced in neutropenic mice or in normal mice treated with neutrophil elastase inhibitors (20). A recent case report described rapid clinical deterioration in an NMO patient made neutrophilic by inadvertent administration of G-CSF (27). Here, we show that eosinophils exacerbate NMO pathology produced by NMO-IgG alone via an ADCC mechanism, or that produced by NMO-IgG and complement via a CDCC mechanism. The approved, nonprescription drugs Zyrtec (cetirizine) and Zaditor (ketotifen) reduced eosinophil-dependent NMO pathology. Our studies suggest the potential clinical utility of granulocyte-targeted therapy with a neutrophil elastase inhibitor together with an eosinophil inhibitor.

Figure 7 diagrams a proposed mechanism for eosinophil-dependent NMO pathology. ADCC is mediated by a variety of leukocyte cell types, including granulocytes, NK cells, and macrophages (28). $\mathrm{ADCC}$ is the principal mechanism in cancer therapy involving the targeting of tumor cells by monoclonal antibodies (29). ADCC involves binding of the antibody Fc region to receptors on effector cells, such as CD16 (FcyRIII) on NK cells, and CD64 (FcyRI), CD32 (Fc $\gamma \mathrm{RII})$, and CD16 on neutrophils and eosinophils (30-32). $\mathrm{CDC}$ is thought to be the major pathogenic mechanism in NMO, based in part on the perivascular deposition of activated complement in human NMO lesions, and the generation of NMO-like pathology in mice administered NMO-IgG and complement. The complement C5 inhibitor eculizumab, which binds to complement protein $\mathrm{C} 5$ and inhibits its cleavage by the C5 convertase, is in an open-label clinical trial based on the assumed dominant role of complement in NMO. The relative importance of ADCC in NMO pathogenesis is not clear. We showed recently that NK cells, acting through a pure ADCC mechanism, produced astro- 
cyte cytotoxicity and NMO-like pathology in mouse models, but without marked inflammation or myelin loss (33). CDCC, which involves inflammatory cell activation by activated complement proteins, has been demonstrated to cause NMO pathology when NMO-IgG and complement are present, together with NK cells, neutrophils or macrophages $(15,33)$, and, as shown here, eosinophils. We thus conclude, as summarized in Figure 7, that NMOIgG produces NMO pathology by a combination of CDC, CDCC, and ADCC mechanisms whose exact contributions may vary over time and remain to be quantified.

Eosinophil degranulation occurs in response to various stimuli including IgG and IgE, and proinflammatory mediators and cytokines such as PAF, fMLP, and complement (12). Proteins contained within eosinophil granules can exacerbate NMO pathology by multiple mechanisms: the granule toxins MBP, ECP, and eosinophil neurotoxins damage adjacent cells directly, and released cytokines and chemokines induce granulocyte and macrophage infiltration (13). Here, we found that even in the absence of NMO-IgG, the release of eosinophil granule contents caused by $\mathrm{C} 5 \mathrm{a}$ or PAF can produce or exacerbate NMO lesions in spinal cord slice cultures.

Cetirizine (Zyrtec) and ketotifen (Zaditor) are clinically approved nonprescription drugs that are long-acting, second-generation histamine $\mathrm{H} 1$ receptor antagonists used in the treatment of atopic dermatitis, urticaria, and allergic rhinitis (34). Both drugs are reported to have multiple actions on eosinophils, including inhibition of eosinophil degranulation, adhesion to endothelial cells, migration in response to AMLP, C5a, and LTB4 chemotaxin, and overall survival (13). The lack of protection by the first-generation cetirizine analogs chlorphenamine, mepyramine, and hydroxyzine indicates that the effects of cetritizine are histamine $\mathrm{H} 1$ receptor independent. Low concentrations of cetirizine increase lipid order in the exterior leaflet of the eosinophil plasma membranes, decrease membrane heterogeneity, and block PAF-induced changes in membrane fluidity (35). Cetirizine was originally designed for minimal brain-blood barrier penetration to reduce the sedative side effect of first-generation antihistamines. Notably, the blood-brain barrier is leaky in NMO lesions, suggesting effective penetration of cetirizine in these lesions. Pharmacokinetic studies showed a mean peak serum cetirizine concentration of $900 \mathrm{ng} / \mathrm{ml}$ (36), similar to its IC $_{50}$ found here for the inhibition of eosinophil EPO release.

Though our data indicate the likely mechanisms by which eosinophil accumulation in NMO lesions produces NMO pathology, it is difficult to quantify the precise contribution of eosinophils in human NMO because of the limitations of available animal models of NMO. While cell cultures are useful for studying the mechanisms of NMO-IgG and AQP4-dependent cytotoxicity, the ex vivo and in vivo models of NMO can recapitulate the major features of human NMO pathology. An important advance here was the development of a continuous intracerebral infusion model that closely recapitulated NMO pathology, including prominent eosinophil and neutrophil infiltration. Nonetheless, as discussed (5), it is difficult to quantitatively extrapolate results from passive transfer models of NMO in rodents to human NMO. Improved animal models of NMO optic neuritis and transverse myelitis are needed, perhaps involving active immunity against AQP4.

In conclusion, our results support the involvement of eosinophils in NMO pathogenesis by ADCC and CDCC mechanisms, which amplify NMO-IgG-dependent astrocyte cytotoxicity and consequent blood-brain barrier disruption and oligodendrocytic injury. Our data thus provide a functional correlate to the prominent eosinophil infiltration in human NMO lesions and suggest the utility of eosinophil-targeted therapeutics in NMO.

\section{Methods}

Materials - mice, cell lines, antibodies, and drugs. AQP4-null mice have been described and extensively characterized (37). Experiments were done using weight-matched 16- to 18 -week-old wild-type and AQP4-null mice on a CD1 genetic background. Some experiments were conducted using hypereosinophilic IL-5 $\operatorname{Tg}(\operatorname{Tg}(C d 3 d-I l 5) N J .1638 N a l)$ mice (38) on a BALB/c background (provided by Richard Locksley, UCSF, San Francisco, California, USA) and $\Delta d b l$ Gata1 mice (C.Cg-Gata $1^{\text {tm } 6 S h o} / J$ mice; The Jackson Laboratory) that lack eosinophils because of Gata1 promoter mutation. Mice were maintained in air-filtered cages and fed normal mouse chow in the UCSF Animal Care Facility. CHO-K1 cells (ATCC CCL-61) were cultured at $37^{\circ} \mathrm{C}$ in $5 \% \mathrm{CO}_{2} / 95 \%$ air in Ham's $\mathrm{F} 12$ medium (Sigma-Aldrich) containing $10 \%$ FBS (Life Technologies) and 1\% penicillin-streptomycin. Stably transfected cells expressing human M23-AQP4 were generated as described (24). Recombinant monoclonal NMO antibody rAb-53 (denoted as NMO-IgG) was generated from a clonally expanded plasma blast population from the cerebrospinal fluid of an NMO patient, as described and previously characterized $(23,39)$. Point mutations (K326W/E333S) were introduced into the IgG1 Fc sequence of the $\mathrm{rAb}-53$ heavy chain to produce antibody $A Q m a b^{A D C C}$, which selectively lacks $A D C C$ effector function (preserving CDC), and AQmab ${ }^{\mathrm{CDC}}(\mathrm{K} 322 \mathrm{~A})$, which selectively lacks CDC effector function (preserving ADCC) (40). NMO-IgG serum was purified from NMO human sera using a Melon Gel IgG Purification Kit (Thermo Scientific) and concentrated using Amicon Ultra Centrifugal Filter Units (Millipore). Ketotifen, cetirizine, loratadine and other chemicals were purchased from Sigma-Aldrich. Anti-IL-5 monoclonal antibody (TRFK-5 clone; BioLegend) was administered intraperitoneally for eosinophil depletion in mice, and anti-Lg6G monoclonal antibody (1A8 clone; BioLegend) for neutrophil depletion (with rat IgG1 as the control antibody).

Eosinophil culture. Bone marrow cells were collected from the femurs and tibiae of wild-type mice (8-12 weeks of age) by flushing the opened bones with $\mathrm{Ca}^{2+}, \mathrm{Mg}^{2+}$-free HBSS (Life Technologies) containing $0.5 \%$ FBS. Red blood cells were lysed in $6 \mathrm{ml}$ of $0.2 \% \mathrm{NaCl}$ followed by the addition of $14 \mathrm{ml}$ of $1.2 \% \mathrm{NaCl}$. After centrifugation, the cells were washed and filtered using a $70-\mu \mathrm{m}$ cell strainer (BD Biosciences). Cells were cultured at $10^{6}$ per milliliter in RPMI 1640 (Life Technologies) containing 20\% FBS, 1\% penicillin-streptomycin, $2 \mathrm{mM}$ glutamine, $25 \mathrm{mM}$ HEPES, $1 \times$ nonessential amino acids, $1 \mathrm{mM}$ sodium pyruvate and $50 \mu \mathrm{M} 2$-mercaptoethanol (Life Technologies), which was supplemented from days 0 through 4 with $100 \mathrm{ng} / \mathrm{ml}$ SCF (PeproTech) and $100 \mathrm{ng} / \mathrm{ml} \mathrm{FLT3-L} \mathrm{(PeproTech).} \mathrm{On} \mathrm{day} \mathrm{4,} \mathrm{SCF} \mathrm{and}$ FLT3-L were replaced by $10 \mathrm{ng} / \mathrm{ml}$ recombinant mouse (rm) IL-5 (PeproTech). On day 8, the cells were transferred to new flasks and maintained in fresh medium supplemented with rmIL-5. Thereafter, on every other day, one-half of the medium was replaced with fresh medium containing rmIL-5, and cell concentration was adjusted to $10^{6}$ per milliliter. Cultures were used for experiments on days 14-16 after incubation with $10 \mathrm{ng} / \mathrm{ml} \mathrm{rm}$ GM-CSF (PeproTech) for 24 hours. Eosinophil purity was greater than $95 \%$ as judged by immunofluorescence using anti-MBPe antibody (provided by the Lee Laboratory, Mayo Clinic, Scottsdale, Arizona, USA).

$C D C C$ and $A D C C$ measurements. Cells were grown in 96-well plates on glass coverslips until confluence. Eosinophils were used as the effector cells. For assay of CDCC, $\mathrm{CHO}$ cells expressing M23-AQP4 were incubated for specified times with $5 \mu \mathrm{g} / \mathrm{ml} \mathrm{NMO}-\operatorname{IgG}$ or control IgG at $37^{\circ} \mathrm{C}$ together with $1 \%$ hc (Innovative Research), with or without eosinophils at an effector/ target cell ratio of 10:1. Calcein-AM and ethidium homodimer (Life Tech- 
nologies) were added to stain live cells green and dead cells red. For assay of ADCC, cells were incubated for 3 hours at $37^{\circ} \mathrm{C}$ with $20 \mu \mathrm{g} / \mathrm{ml} \mathrm{NMO}-\mathrm{IgG}$ (or control $\operatorname{IgG}$ ) and eosinophils at an effector/target cell ratio of 30:1. For assay of CDC, cells were incubated for 60 minutes at $37^{\circ} \mathrm{C}$ with $10 \mu \mathrm{g} / \mathrm{ml}$ NMO-IgG and $2 \%$ hc. In some experiments, test compounds were added for 30 minutes, followed 60 minutes later by complement and NMO-IgG. Cytotoxicity was measured by the AlamarBlue Assay (Life Technologies).

Spinal cord slice culture model. On postnatal day 7 mouse pups were decapitated and the spinal cord was rapidly removed and placed in ice-cold HBSS. Transverse slices of cervical spinal cord of 300- $\mu$ m thickness were cut using a vibratome (VT-1000S; Leica). Individual slices were placed on transparent, noncoated membrane inserts (Millicell-CM $0.4 \mu \mathrm{m}$ pores, $30-\mathrm{mm}$ diameter; Millipore) in 6-well (35-mm diameter) plates containing $1 \mathrm{ml}$ culture medium, with a thin film of culture medium covering the slices. The culture medium, consisting of 50\% MEM (Life Technologies), 25\% HBSS, 25\% horse serum (Life Technologies), $1 \%$ penicillin-streptomycin, $0.65 \%$ glucose, and $25 \mathrm{mM}$ HEPES, was changed every 3 days. The slices were cultured in $5 \% \mathrm{CO}_{2}$ at $37^{\circ} \mathrm{C}$ for 7 days. NMO-IgG and/or complement were added to both sides of the porous membrane, with $1 \mathrm{ml}$ of medium added above the porous filter to fully immerse the slice. Eosinophils were added to the solution above the porous filter bathing the slice. In some experiments PAF (Sigma-Aldrich), ECP (Sigma-Aldrich), or recombinant human C5a, together with cytochalasin B (Sigma-Aldrich), were added directly to the slice medium for 24 hours. AQP4- and GFAP-stained spinal cord slices were scored for lesion severity using the following scale: 0 , intact slice with intact GFAP and AQP4 staining; 1, intact slice with some astrocyte swelling (seen from GFAP stain) with weak AQP4 staining; 2 , at least 1 lesion with complete loss of GFAP and AQP4 staining; 3, multiple lesions with loss of GFAP and AQP4 staining in greater than $30 \%$ of slice area; and 4 , extensive loss of GFAP and AQP4 staining affecting greater than $80 \%$ of the slice area (15).

Intracerebral infusion model. Mice were implanted with an osmotic minipump (Alzet 1003D) with the needle positioned in the right lateral brain (from the bregma, anterior-posterior, $-2 \mathrm{~mm}$; medial-lateral, $+1.5 \mathrm{~mm}$; dorsal-ventral, $2.5 \mathrm{~mm}$ below the dura). NMO-IgG and he were dissolved in PBS and delivered for 3 days at $3.3 \mu \mathrm{g}$ per day and $16.7 \mu \mathrm{l}$ per day, respectively. For the IL-5 Tg mice, submaximal human complement (3.4 $\mu \mathrm{l}$ per day) was delivered together with NMO-IgG at $3.3 \mu$ g per day. In some studies, purified IgG from human NMO sera was delivered for 3 days at $66 \mu \mathrm{g}$ per day. Control mice were implanted identically with an osmotic minipump with control (non-AQP4-binding) IgG1.

Immunofluorescence and immunochemistry. Slices were fixed with $4 \%$ paraformaldehyde for 15 minutes at room temperature, rinsed with PBS, and incubated in blocking solution (PBS containing 1\% BSA and 0.3\% Triton $\mathrm{X}-100)$ for 1 hour. Slices were then incubated for 2 hours with antibodies against mouse GFAP (1:1000; Millipore), AQP4 (1:200; Millipore), ionized calcium-binding adaptor molecule 1 (Iba1; 1:1,000; Wako), C5b-9 (1:100; Santa Cruz Biotechnology), or MBP (1:100; Santa Cruz Biotechnology), rinsed in PBS, and then incubated with secondary antibodies for 1 hour at room temperature. Secondary antibodies included Alexa Fluor 488-labeled donkey anti-mouse IgG, Alexa Fluor 555-labeled donkey anti-goat IgG, Alexa Fluor 555-labeled donkey anti-rabbit IgG, and Alexa Fluor 555-labeled goat anti-rat IgG ( $5 \mu \mathrm{g} / \mathrm{ml}$ each; Invitrogen). After rinsing in PBS, the slices were mounted on glass slides and coverslipped with VectaShield (Vector Laboratories). For the in vivo mouse model, frozen sections of brain were cut at $10-\mu \mathrm{m}$ thickness and immunostained with the following primary antibodies at room temperature for 2 hours: rabbit anti-AQP4 (1:200); mouse anti-GFAP
(1:500); goat anti-MBP (1:200); rabbit anti-Iba1 (1:500); rat anti-CD45 (1:10; BD Biosciences); rat anti-neutrophil (Ly6G antibody; 1:100; eBioscience); rat anti-eosinophil (Siglec F antibody; 1:50; BD Biosciences); mouse anti-EAAT1/ GLAST (1:100; Miltenyi Biotec); or rabbit anti-C5b-9 (1:50; Santa Cruz Biotechnology), followed by the appropriate species-specific Alexa Fluor-conjugated secondary antibody $(5 \mu \mathrm{g} / \mathrm{ml}$ each) or biotinylated secondary antibody (1:500; Vector Laboratories). When biotinylated secondary antibody was used, immunostaining was visualized as brown using the Vectastain horseradish peroxidase kit (Vector Laboratories), followed by diaminobenzidine $/ \mathrm{H}_{2} \mathrm{O}_{2}$ according to the manufacturer's protocol. Immunofluorescence was visualized on a Leica fluorescence microscope or a Nikon laser-scanning confocal microscope. Areas were defined by hand and quantified using ImageJ software (NIH). Data are presented as the percentage of immunonegative area (normalized to the total area of the hemibrain section).

Eosinophil peroxidase release activity. Release of eosinophil peroxidase was measured in cell supernatants by the o-phenylenediamine (OPD) method (41). Substrate solution was prepared by adding $800 \mu \mathrm{l}$ of $5 \mathrm{mM} \mathrm{OPD} \mathrm{HCl}$ (Sigma-Aldrich) to $4 \mathrm{ml}$ of $1 \mathrm{M}$ Tris buffer ( $\mathrm{pH}$ 8.0) and $1.25 \mu \mathrm{l} 30 \% \mathrm{H}_{2} \mathrm{O}_{2}$. Eosinophils were collected by centrifugation and resuspended in RPMI 1640 , without phenol red, at 250,000 cells per milliliter and $100 \mu \mathrm{l}$ was added per well. EPO release from eosinophils was measured following the addition of PAF, which causes eosinophil degranulation (41). Substrate solution $(100 \mu \mathrm{l})$ was added after 30 minutes, and the reaction stopped by $100 \mu \mathrm{l}$ of $4 \mathrm{M}$ sulfuric acid prior to the measurement of absorbence at 492 $\mathrm{nm}$. In some experiments, eosinophil degranulation inhibitors (cetirizine, ketorizine, loratadine, cromolyn sodium, theophylline, IBMX) were added (separately) 30 minutes before PAF. EPO release was also measured after ADCC in which 250,000 cells in $100 \mu$ l were added to 96-well plates containing cultured CHO-AQP4 cells or CHO-null cells. NMO-IgG or control IgG was added and cells were incubated at $37^{\circ} \mathrm{C}, 5 \% \mathrm{CO}_{2}$ for 1 hour. Samples were centrifuged at $250 \mathrm{~g}$ for 15 minutes at $4^{\circ} \mathrm{C}$. The supernatants (50 $\mu \mathrm{l}$; triplicate) were dispensed into each well of a microplate, and $50 \mu \mathrm{l}$ of substrate solution was added to each well.

Statistics. Where appropriate, differences were evaluated using a 2-tailed Student's $t$ test, a Mann-Whitney rank sum test, or a 1-way ANOVA rank test, as calculated using SigmaStat software, version 3.5 (Systat Software). Data are presented as the mean \pm SEM. A $P$ value of 0.05 or less was considered significant.

Study approval. All mouse procedures were approved by the University of California, San Francisco Committee on Animal Research and were conducted in accordance with the NIH guidelines for the care and use of animals.

\section{Acknowledgments}

This work was supported by a grant from the Guthy-Jackson Charitable Foundation, and by grants from the NIH (EY13574, EB00415, DK35124, HL73856, DK86125, and DK72517). We thank Jeffrey Bennett of the University of Colorado Denver for providing recombinant monoclonal NMO antibody and for helpful advice, and Puay-Wah Phuan for help with CDC measurements.

Received for publication October 26, 2012, and accepted in revised form February 12, 2013.

Address correspondence to: Alan S. Verkman, 1246 Health Sciences East Tower, University of California, San Francisco, California 94143-0521, USA. Phone: 415.476.8530; Fax: 415.665.3847; E-mail: Alan.Verkman@ucsf.edu.
1. Jarius S, et al. Mechanisms of disease: aquaporin-4 antibodies in neuromyelitis optica. Nat Clin Pract Neurol. 2008;4(4):202-214.

2. Wingerchuk DM, et al. The spectrum of neuromy- elitis optica. Lancet Neurol. 2007;6(9):805-815.

3. Lennon VA, Kryzer TJ, Pittock SJ, Verkman AS, Hinson SR. IgG marker of optic-spinal multiple sclerosis binds to the aquaporin-4 water channel.
JExp Med. 2005;202(4):473-477.

4. Jarius S, Wildemann B. AQP4 antibodies in neuromyelitis optica: diagnostic and pathogenetic relevance. Nat Rev Neurol. 2010;6(7):383-392. 
5. Papadopoulos MC, Verkman AS. Aquaporin 4 and neuromyelitis optica. Lancet Neurol. 2012; 11(6):535-544

6. Misu T, et al. Loss of aquaporin 4 in lesions of neuromyelitis optica: distinction from multiple sclerosis. Brain. 2007;130(pt 5):1224-1234.

7. Lucchinetti CF, et al. A role for humoral mechanisms in the pathogenesis of Devic's neuromyelitis optica. Brain. 2002;125(pt 7):1450-1461.

8. Hengstman GJ, Wesseling P, Frenken CW, Jongen PJ. Neuromyelitis optica with clinical and histopathological involvement of the brain. Mult Scler. 2007;13(5):679-682.

9. Popescu BF, et al. Neuromyelitis optica unique area postrema lesions: nausea, vomiting, and pathogenic implications. Neurology. 2011;76(14):1229-1237.

10. Cree BA, Lamb S, Morgan K, Chen A, Waubant E, Genain C. An open label study of the effects of rituximab in neuromyelitis optica. Neurology. 2005 64(7):1270-1272.

11. Miyamoto K, Kusunoki S. Intermittent plasmapheresis prevents recurrence in neuromyelitis optica. Ther Apher Dial. 2009;13(6):505-508.

12. Rothenberg ME, Hogan SP. The eosinophil. Annu Rev Immunol. 2006;24:147-174.

13. Giembycz MA, Lindsay MA. Pharmacology of the eosinophil. Pharmacol Rev. 1999;51(2):213-340.

14. Fulkerson PC, Rothenberg ME. Targeting eosinophils in allergy, inflammation and beyond. Nat Rev Drug Discov. 2013;12(2):117-129.

15. Zhang H, Bennett JL, Verkman AS. Ex vivo spinal cord slice model of neuromyelitis optica reveals novel immunopathogenic mechanisms. Ann Neurol. 2011;70(6):943-954.

16. Dyer KD, Moser JM, Czapiga M, Siegel SJ, Percopo CM, Rosenberg HF. Functionally competent eosinophils differentiated ex vivo in high purity from normal mouse bone marrow. I Immunol. 2008 181(6):4004-4009.

17. Stolarski B, Kurowska-Stolarska M, Kewin P, Xu D, Liew FY. IL-33 exacerbates eosinophilmediated airway inflammation. J Immunol. 2010; 185(6):3472-3480.

18. Ezeamuzie CI, Taslim N. Protein kinase C activation inhibits eosinophil degranulation through stimulation of intracellular cAMP production. $\mathrm{Br}$ J Pharmacol. 2004;143(6):725-732.
19. Saadoun S, Waters P, Bell BA, Vincent A, Verkman AS, Papadopoulos MC. Intra-cerebral injection of neuromyelitis optica immunoglobulin G and human complement produces neuromyelitis optica lesions in mice. Brain. 2010;133(pt 2):349-361.

20. Saadoun $S$, et al. Neutrophil protease inhibition reduces neuromyelitis optica-immunoglobulin G-induced damage in mouse brain. Ann Neurol. 2012; 71(3):323-333.

21. Yamada T, Tani Y, Nakanishi H, Taguchi R, Arita $\mathrm{M}$, Arai $\mathrm{H}$. Eosinophils promote resolution of acute peritonitis by producing proresolving mediators in mice. FASEB J. 2011;25(2):561-568.

22. Matsumori A, Yamamoto K, Shimada M. Cetirizine a histamine $\mathrm{H} 1$ receptor antagonist improves viral myocarditis. J Inflamm (Lond). 2010;7:39.

23. Crane JM, Lam C, Rossi A, Gupta T, Bennett JL, Verkman AS. Binding affinity and specificity of neuromyelitis optica autoantibodies to aquaporin-4 M1/ M23 isoforms and orthogonal arrays. J Biol Chem. 2011;286(18):16516-16524.

24. Phuan PW, Ratelade J, Rossi A, Tradtrantip L, Verkman AS. Complement-dependent cytotoxicity in neuromyelitis optica requires aquaporin-4 protein assembly in orthogonal arrays. J Biol Chem. 2012; 287(17):13829-13839.

25. Rossi A, Moritz TJ, Ratelade J, Verkman AS. Super-resolution imaging of aquaporin- 4 orthogonal arrays of particles in cell membranes. J Cell Sci. 2012;125(18):4405-4412

26. Rossi A, Ratelade J, Papadopoulos MC, Bennett JL, Verkman AS. Neuromyelitis optica IgG does not alter aquaporin-4 water permeability, plasma membrane M1/M23 isoform content, or supramolecular assembly. Glia. 2012;60(12):2027-2039.

27. Jacob A, et al. Detrimental role of granulocytecolony stimulating factor in neuromyelitis optica: clinical case and histological evidence. Mult Scler. 2012;18(12):1801-1803.

28. Capel PJ, van de Winkel JG, van den Herik-Oudijk IE, Verbeek JS. Heterogeneity of human IgG FC receptors. Immunomethods. 1994;4(1):25-34.

29. Shuptrine CW, Surana R, Weiner LM. Monoclonal antibodies for the treatment of cancer. Semin Cancer Biol. 2012;22(1):3-13.

30. Hubert P, et al. Antibody-dependent cell cytotoxicity synapses form in mice during tumor-specific antibody immunotherapy. Cancer Res. 2011;71(15):5134-5143.

31. Siders WM, et al. Involvement of neutrophils and natural killer cells in the anti-tumor activity of alemtuzumab in xenograft tumor models. Lenk Lymphoma. 2010;51(7):1293-1304.

32. Valerius T, Repp R, Kalden JR, Platzer E. Effects of IFN on human eosinophils in comparison with other cytokines. A novel class of eosinophil activators with delayed onset of action. J Immunol. 1990; 145(9):2950-2958.

33. Ratelade J, Zhang H, Saadoun S, Bennett JL, Papadopoulos MC, Verkman AS. Neuromyelitis optica IgG and natural killer cells produce NMO lesions in mice without myelin loss. Acta Neuropathol. 2012;123(6):861-872

34. Spencer CM, Faulds D, Peters DH. Cetirizine. A reappraisal of its pharmacological properties and therapeutic use in selected allergic disorders. Drugs. 1993;46(6):1055-1080.

35. Kantar A, Oggiano N, Giorgi PL, Rihoux JP. A study of the interaction between cetirizine and plasma membrane of eosinophils, neutrophils, platelets and lymphocytes using a fluorescence technique. Mediators Inflamm. 1994;3(3):229-234.

36. Watson WT, Simons KJ, Chen XY, Simons FE. Cetirizine: a pharmacokinetic and pharmacodynamic evaluation in children with seasonal allergic rhinitis. J Allergy Clin Immunol. 1989;84(4 pt 1):457-464.

37. Ma T, Yang B, Gillespie A, Carlson EJ, Epstein CJ, Verkman AS. Generation and phenotype of a transgenic knockout mouse lacking the mercurial-insensitive water channel aquaporin-4.J Clin Invest. 1997;100(5):957-962.

38. Wu D, et al. Eosinophils sustain adipose alternatively activated macrophages associated with glucose homeostasis. Science. 2011;332(6026):243-247.

39. Bennett JL, et al. Intrathecal pathogenic antiaquaporin-4 antibodies in early neuromyelitis optica. Ann Neurol. 2009;66(5):617-629.

40. Tradtrantip L, et al. Anti-aquaporin-4 monoclonal antibody blocker therapy for neuromyelitis optica. Ann Neurol. 2012;71(3):314-322.

41. Dyer KD, et al. Mouse and human eosinophils degranulate in response to platelet-activating factor (PAF) and lysoPAF via a PAF-receptor-independent mechanism: evidence for a novel receptor.J Immunol. 2010;184(11):6327-6334. 